\title{
The future of the high energy cosmic ray detection: HERD
}

\author{
Chiara Perrina ${ }^{1, a}$ on behalf of the HERD Collaboration \\ ${ }^{1}$ DPNC, Université de Genève, 24 quai Ernest-Ansermet, $\mathrm{CH}-1211$ Genève 4, Switzerland
}

\begin{abstract}
The High Energy cosmic-Radiation Detection (HERD) facility will be one of the space astronomy payloads on board the future Chinese space station. The ambitious aim of HERD is the direct detection of cosmic rays towards the "knee" region $(\sim 1 \mathrm{PeV})$, with a detector able to measure electrons, photons and nuclei with an excellent energy resolution ( $1 \%$ for electrons and photons at $200 \mathrm{GeV}$ and $20 \%$ for nuclei at $100 \mathrm{GeV}-\mathrm{PeV}$ ), an acceptance 10 times the one of present generation missions $\left(\sim 1 \mathrm{~m}^{2} \mathrm{sr}\right)$, and long lifetime ( $>10$ years). The primary objectives of HERD are the indirect search for dark matter particles and the precise measurement of energy distribution and composition of cosmic rays from $30 \mathrm{GeV}$ up to a few $\mathrm{PeV}$, determining the origin of the "knee" structure of the spectrum. Furthermore, HERD will monitor the high energy gamma-ray sky from $500 \mathrm{MeV}$, observing gamma-ray bursts, active galactic nuclei, galactic microquasars, etc. HERD will be composed of a homogeneous calorimeter, surrounded by a particle tracker and a plastic scintillator detector. Two possible trackers are under study: a 5-side tracker made of silicon strip detectors and a 4-side scintillating fiber tracker with a silicon strip top tracker. The total volume of HERD will be $(2.3 \times 2.3 \times 2.6) \mathrm{m}^{3}$ with a weight of about $4 \mathrm{t}$. The HERD design, perspectives, expected performances in terms of energy sensitivity and acceptance will be presented in this contribution.
\end{abstract}

\section{Introduction}

From 2025, the expected launch year, and for at least 10 years, HERD will be observing the high energy sky from the Chinese space station (CSS). Thanks to its unprecedented geometric acceptance at $\mathrm{PeV}$ energies, HERD represents the future of the direct high energy cosmic ray detection. An international scientific collaboration is growing around the HERD project: at present, it involves researchers from Chinese institutions lead by CSU and IHEP, Italy, Switzerland and Spain, but more institutes have shown interest and will eventually join the effort.

\section{The objectives of HERD}

The main objectives of HERD are:

1. The search for signatures of annihilation and decay products of dark matter (DM) both in the energy spectrum and in anisotropy of high energy electrons from $10 \mathrm{GeV}$ to $100 \mathrm{TeV}$ and in the gamma-ray spectrum from $500 \mathrm{MeV}$ to $100 \mathrm{TeV}$. HERD will have an unprecedented sensitivity

\footnotetext{
ae-mail: chiara.perrina@unige.ch
} 
Table 1. Main requirements for the HERD payload

\begin{tabular}{lc}
\hline Energy range $(\mathrm{e} / \gamma)$ & $10 \mathrm{GeV}-100 \mathrm{TeV}$ \\
Low energy range for $\gamma$ & $500 \mathrm{MeV}-10 \mathrm{GeV}$ \\
Energy range (nuclei) & $30 \mathrm{GeV}-3 \mathrm{PeV}$ \\
\hline Angular resolution $(\mathrm{e} / \gamma)$ & $0.1^{\circ}$ at $10 \mathrm{GeV}$ \\
Charge resolution $($ nuclei) & $10 \%-15 \%$ for $Z=1-26$ \\
\hline Energy resolution $(\mathrm{e} / \gamma)$ & $<1 \%$ at $200 \mathrm{GeV}$ \\
Energy resolution $(\mathrm{p})$ & $20 \%$ at $100 \mathrm{GeV}-\mathrm{PeV}$ \\
e/p separation power & $>10^{5}$ \\
Geometric factor $(\mathrm{e})$ & $>3 \mathrm{~m}^{2} \mathrm{sr}$ at $200 \mathrm{GeV}$ \\
Geometric factor $(\mathrm{p})$ & $>2 \mathrm{~m}^{2} \mathrm{sr}$ at $100 \mathrm{GeV}$ \\
\hline
\end{tabular}

in searching for DM signals thanks to its large geometric acceptance, excellent energy resolution and long duration. The current generation of missions such as AMS-02 [1] and DAMPE [2] have observed some unexpected features in the positron fraction and in the total electron spectrum that could be related to DM, but could also be produced by nearby pulsars. HERD will perform high statistics and precise measurements in the same energy range and beyond, to more than $10 \mathrm{TeV}$, of both spectral features and anisotropy, which will definitely resolve the question whether the features are due to nearby sources. Fig. 1 (left) illustrates the expected HERD capability to distinguish if the excess in the total electron flux $\left(\mathrm{e}^{-}+\mathrm{e}^{+}\right)$is due to an astrophysical source, like a pulsar wind nebula (PWN), or to DM. Another particularly interesting detection channel is provided by $\gamma$-rays. Two-body DM annihilation or DM decay could be observed as a sharp peak over the high energy diffuse $\gamma$-ray background. The $\gamma$-ray line feature is considered as the "smoking gun" signal of DM. HERD will cover the $\gamma$-ray line searches from $10 \mathrm{GeV}$ to $10 \mathrm{TeV}$ with the best sensitivity ever achieved.

2. The measurement of energy spectrum and composition of cosmic rays (protons and nuclei) from $30 \mathrm{GeV}$ to a few $\mathrm{PeV}$ that carry the information on the production, acceleration and propagation of these particles, which is fundamental to understand the formation and the evolution of stars and galaxies, and the extreme energetic processes such as supernova explosions, pulsar jets, and neutron star and black hole merging. HERD will measure the spectra of different chemical elements in cosmic rays, from proton to iron, with an unprecedented precision, up to the "knee" region where ground-based experiments have observed a change of spectral index in the allparticle cosmic ray flux, which could indicate the confinement limit of high energy particles by the galactic magnetic field, and the existence of extra-galactic sources. HERD will perform these measurements directly in space, avoiding large systematic effects associated with groundbased experiment, thus helping to elucidate another great puzzle in cosmic ray physics. Fig. 1 (center) shows the simulation of the proton flux as measured by HERD after 5 years.

3. A wide field of view monitoring of $\gamma$-rays from $500 \mathrm{MeV}$ to study gamma-ray bursts, active galactic nuclei and galactic microquasars. The monitoring of high energy $\gamma$-ray sources in space is very crucial in the era of the multi-messenger and multi-wavelength astronomy, as has been demonstrated by the recent detection by Fermi [3] of the counterpart of the gravitational wave from a neutron star merger detected by LIGO. HERD will play a dominant role in the GeV-TeV $\gamma$-ray astronomy in 2025-2035. Without HERD there will be a large gap in the coverage of the electromagnetic spectrum. The sky map expected from HERD after 1 year of activity is illustrated in Fig. 1 (right). 

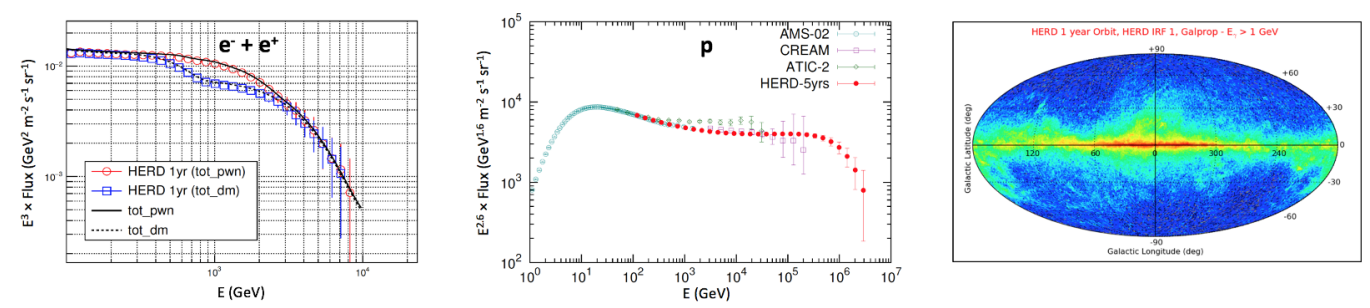

Figure 1. Expected $\mathrm{e}^{-}+\mathrm{e}^{+}$flux as measured by HERD with one year data accumulation. HERD will clearly distinguish between a smooth spectrum, due to a PWN, or a spectrum with a "kink", due to DM (left). Expected proton flux as measured by HERD with five years data accumulation. HERD can reach the PeV region with unprecedented statistics (center). Expected HERD's one-year map of the gamma-ray sky (right).

In Table 1 the requirements for HERD in terms of energy range, energy, angular and charge resolution, electron/proton separation and geometric factors are listed. Moreover it is important to note that the geometric factors are $\sim 10$ times the geometric factors of present detectors, with a field of view of $\pm 70^{\circ}$.

\section{The HERD detector}

Fig. 2 shows the exploded view of the HERD detector in which it is possible to see the different sub-detectors. From inside to outside, the HERD detector consists of a calorimeter (CALO) for the measurement of the energy of traversing particles and for the electrons/protons discrimination, a 5-side particle tracker which will serve to reconstruct the particle trajectories and to identify the nuclei charge, a plastic scintillator detector (PSD) for the measurement of low energy $\gamma \mathrm{s}$ and for a redundant measurement of the nuclei charge and a transition radiation detector (TRD) to perform the energy calibration. The option with a 4-side scintillating fiber tracker (FIT) and a top silicon strip tracker (STK) is depicted.

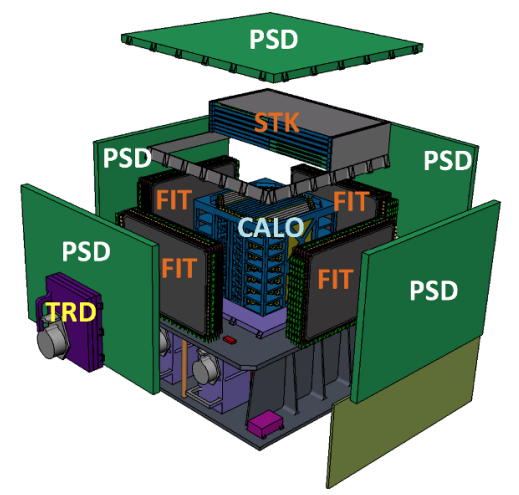

Figure 2. Exploded view of the HERD detector. The whole apparatus will have a weight $<4 \mathrm{t}$ and a volume of $(2.3 \times 2.3 \times 2.6) \mathrm{m}^{3}$.

\subsection{The calorimeter (CALO)}

The calorimeter comprises three sub-systems: the crystal array, the intensified scientific CMOS (IsCMOS) camera and the trigger sub-system. The crystal array (Fig. 3 left) is made of about 7500 cubic 
crystals with $3 \mathrm{~cm}$ side, corresponding to $\sim 55$ radiation lengths and $\sim 3$ nuclear interaction lengths in all directions. Cosmic ray measurements up to PeV energy is then made feasible with $20 \%$ energy resolution and an e/p separation power of at least $10^{5}$. A novel method of reading the LYSO signals by wavelength shifting fibers (WLSFs) and IsCMOS is adopted for its compactness, simple electronics and external power consumption. For the realization of larger dynamic range, each crystal is coupled with three WLSFs, reshaped into spirals (Fig. 3 center) to get the largest contact area with the crystal. Two of them are for high and low range IsCMOS cameras and the other one for the trigger sub-system. The crystal and fibers are coated with reflectors to increase light output (Fig. 3 right). The complete MIP peak shall be visible in the spectrum: the starting point of the dynamic range of the camera is $1 / 3 \mathrm{MIP}(1 \mathrm{MIP} \simeq 30 \mathrm{MeV}$ in a LYSO cube), while the maximum energy deposition in one crystal, calculated on simulation, is about $20 \mathrm{TeV}$ which is created by a PeV hadronic shower. Therefore the dynamic range of the readout system should be about $10^{7}$, thus two different image intensifiers with two different gain settings are needed. All the 7500 trigger fibers are routed to the PMTs of the trigger sub-system, dedicated to providing common trigger signals to all the other instruments after veto and coincidence logic. A beam test of a HERD calorimeter prototype consisting of 250 LYSO crystals was conducted at the CERN SPS H2 and H4 beam lines in 2017. Preliminary results show the good energy linearity of the calorimeter. The energy resolution is better than $1.3 \%$ for $200 \mathrm{GeV} / \mathrm{c}$ electrons.
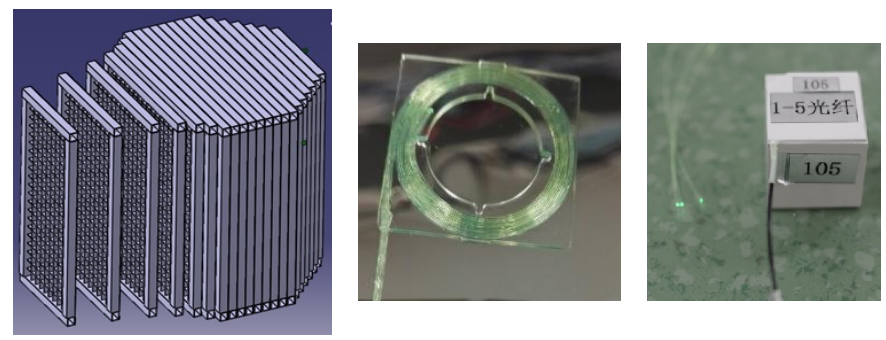

Figure 3. Sketch of the crystal array of the HERD calorimeter. It will consist of 21 layers of cubic LYSO crystals with $3 \mathrm{~cm}$ sides (left). Three WLSFs reshaped into spirals are coupled to one side of the crystal (center). The crystal sides are covered with reflectors to increase the light output (right).

\subsection{The tracking detector}

Two possible tracker options are under study: a 5-side tracker made of silicon strip detectors (SSDs) and a 4-side scintillating fiber tracker with a silicon strip tracker on the top.

\subsubsection{STK}

The Silicon Tracker (STK) is designed to perform particle track reconstruction and particle charge measurement up to $Z=26$. The top STK (Fig. 4 panel 2) is composed of six double planes of silicon detectors, each plane has an active area of $133 \mathrm{~cm} \times 133 \mathrm{~cm}$ and is equipped with 28 Type A ladders made of 7 single-sided SSDs each. Ladders on alternate planes are placed orthogonally in order to measure the $\mathrm{x}$ and $\mathrm{y}$ coordinate of the incoming particle. Lateral STKs are composed of three positionsensitive double planes of silicon detectors. Each plane has an active area of $95 \mathrm{~cm} \times 66.5 \mathrm{~cm}$ and is equipped with 10 Type A ladders (Fig. 4 panel 3) or 14 Type B ladders (Fig. 4 panel 4). Each Type B ladder is assembled with 5 single sided SSDs. The material between the active parts will be minimized to ensure the physics performance while keeping the stability of the overall mechanics. The SSD is the key component in STK and the HERD baseline is to use the same SSD as in DAMPE [4] (Fig. 4 
panel 1). With a strip pitch of $121 \mu \mathrm{m}$ and a readout pitch of $242 \mu \mathrm{m}$ we expect a resolution of about $40 \mu \mathrm{m}$ after proper alignment. The readout of one every other strip (corresponding to 384 channels per ladder) is a balance among a good performance in terms of spatial resolution and a limited power consumption.
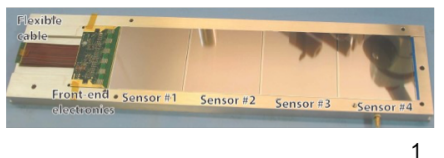

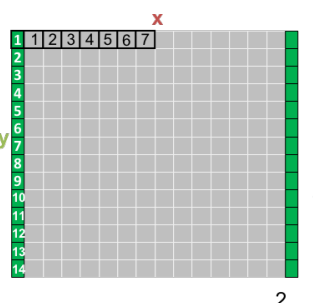

2

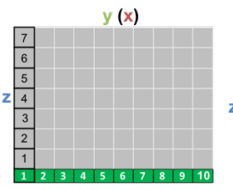

3

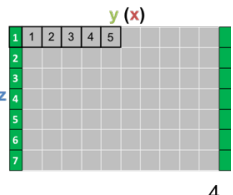

4

Figure 4. DAMPE ladder equipped with 4 SSDs made by Hamamatsu with an area of $95 \mathrm{~mm} \times 95 \mathrm{~mm}(1)$. Sketch of a top STK plane equipped with 28 Type A ladders (2). Sketch of a lateral STK plane equipped with 10 Type A ladders (3). Sketch of a lateral STK plane equipped with 14 Type B ladders (4).

\subsubsection{FIT}

For the lateral STK, the requirement of a full acceptance puts very stringent constraints on the placement of front-end and readout electronics. For this reason a scintillating fiber tracker provides a very interesting alternative to a silicon strip tracker since fiber mats can be easily adapted to the needed geometry. There are several motivations to choose a scintillating fiber tracker: it would be simpler to build such a long detector $(O(1 \mathrm{~m})$ ), the dead area would be reduced (no gap between sensors, no dead area on the silicon detector, front-end and readout electronics placed outside of support trays), it would be less expensive to add more layers, thus improving the point spread function of low energy photons and more robust because wire bonds on the detectors are not needed.
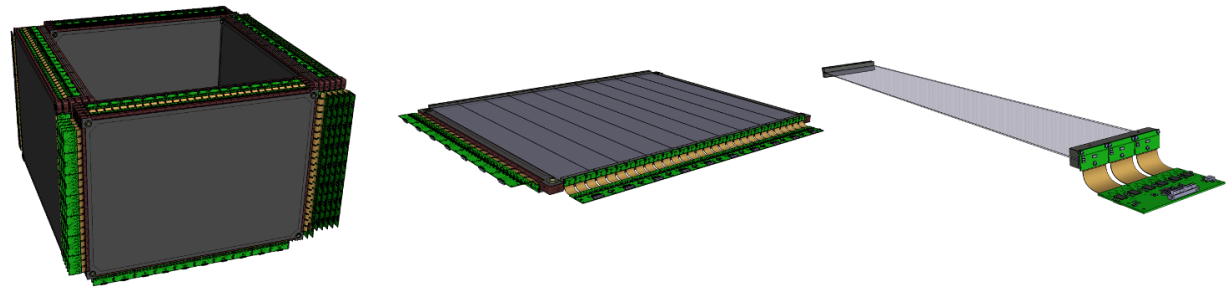

Figure 5. Sketch of the 4-side FIT detector (left). Sketch of one x-y plane (center). Sketch of a FIT module (right).

According to the current design, the FIT will be composed of four identical sectors (Fig. 5 left) equipped with $5 \mathrm{x}$-y planes (Fig. 5 center). A x plane is made of $7 \mathrm{x}$-modules, a y plane of 10 y-modules. The module is the elementary unit of the FIT detector (Fig. 5 right). It includes a fiber mat and 3 silicon photomultiplier (SiPM) arrays to read-out the scintillation light. The overall mass will be $\sim 250 \mathrm{~kg}$, the overall dimensions $(1.4 \times 1.4 \times 0.9) \mathrm{m}^{3}$. The fiber mat has two possible lengths: $1.06 \mathrm{~m}$ for $\mathrm{x}$-modules and $77 \mathrm{~cm}$ for y-modules, and a width of $97.8 \mathrm{~mm}$ to match $3 \mathrm{SiPM}$ arrays. The mat is made of 6 layers of fibers. The used fibers are made by Kuraray (SCSF-78MJ type), they have a round section with a diameter of $250 \mu \mathrm{m}$ and the peak emission is at $450 \mathrm{~nm}$ wavelength. Except for lenght and width, the fiber mat is the same used for LHCb fiber tracker upgrade [5]. Also the SiPM arrays so far used for tests are the same used for LHCb experiment: S133552-HRQ type, 
made by Hamamatsu with 2 chips/array and 64 channels/chip. Each channel contains $4 \times 26$ pixels of $57.5 \mu \mathrm{m} \times 62.5 \mu \mathrm{m}$ area, with a gain of $10^{6}$. A different SiPM array produced by Hamamatsu for the University of Geneva is under test. This is the S13552-10 type with 2 chips of 64 channels, customised with $23 \times 163$ pixels/channel of $10 \mu \mathrm{m} \times 10 \mu \mathrm{m}$. The latter would assure the measurement of charged particles up to at least $Z=19$. A beam test of a FIT module prototype consisting of a $1 \mathrm{~m}$ fiber mat coupled to one S133552-HRQ SiPM array was conducted at the CERN SPS H2 beam line in 2017. Preliminary results for $100 \mathrm{GeV}$ pions show a nominal light yield of 16 photoelectrons (Fig. 6 left), a good spatial resolution of $59.6 \mu \mathrm{m}$ (Fig. 6 right, $70 \mu \mathrm{m}$ would be sufficient for our application because it guarantees an angular resolution of $0.1^{\circ}$ ) and a hit efficiency of $99.7 \%$.
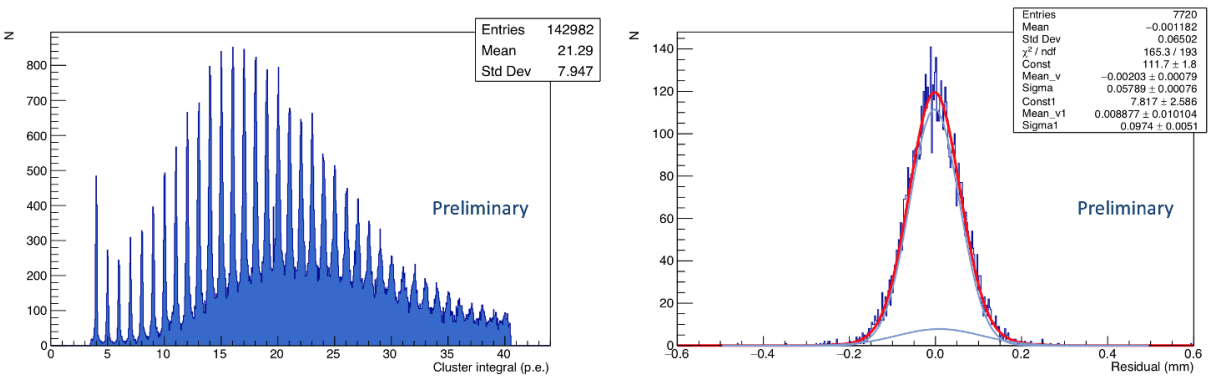

Figure 6. Beam test results for a FIT prototype module made of $1 \mathrm{~m}$ length fiber mat and one SiPM array: light yield (left) and hit residual distribution with double Gaussian fits (right).

An option to use $10 \mathrm{x}-\mathrm{y}$ planes for the fiber tracker has been proposed to provide unprecedented sensitivity to gamma-rays of $100 \mathrm{MeV}$ to a few $\mathrm{GeV}$.

\section{The role of HERD in the multi-messenger astronomy}

HERD with its large field of view and unique energy coverage will play a unique and complementary role in multi-wavelength studies across the electromagnetic spectrum with other space and ground telescopes involving radio, optical, $\mathrm{X}$-ray, $\gamma$-ray, and in the search for the electromagnetic counterpart of gravitational waves and for contemporaneous flaring in photon data and neutrinos (IceCube, KM3Net). Thanks to the trio HERD + CTA + LHAASO there will be a simultaneous coverage of the same sources from a few $\mathrm{GeV}$ to a few $\mathrm{PeV}$, in addition to an overlap of measured spectra. It will be possible to distinguish diffuse emission from localized contributions, to disentangle acceleration and propagation mechanism in SNRs, PWNe, pulsars, and in more extended objects as the Fermi bubbles, to study transient phenomena, which is crucial to analyse the properties of jets, and can help determining the extragalactic background light, intergalactic magnetic fields, and the validity of the Lorentz invariance. Moreover, HERD will produce ToO alerts for AGN, novae, binary systems.

\section{Summary and outlook}

HERD will operate on the CSS starting from 2025 for more than 10 years. It will be a calorimetric detector with unprecedented acceptance. It will have important and frontier scientific objectives in dark matter search, cosmic ray physics and gamma-ray astronomy. HERD could become the only space-borne high energy $\gamma$-ray detector, once the Fermi satellite will stop its operations. The baseline detector is defined and fulfils the requirements. To optimise the detector geometry a dedicated Monte Carlo simulation is being developed, based on the Geant4 framework. Several studies for further improvements and optimization are ongoing. 


\section{References}

[1] M. Aguilar et al. (AMS), Phys. Rev. Lett. 113, 221102 (2014)

[2] G. Ambrosi et al. (DAMPE), Nature 552, 63 (2017), 1711. 10981

[3] M. Ajello et al. (Fermi-LAT), Astrophys. J. 861, 85 (2018)

[4] A. Tykhonov et al. (DAMPE), Nucl. Instrum. Meth. A893, 43 (2018), 1712.02739

[5] P. Hopchev (LHCb SciFi Tracker), SciFi: A large Scintillating Fibre Tracker for LHCb, in 5th Large Hadron Collider Physics Conference (LHCP 2017) Shanghai, China, May 15-20, 2017 (2017), 1710.08325 